1873 m. Berl. Zt. $\quad \alpha 1873.0$ Juli $\quad 6.5 \quad 4^{0} \quad 2^{\prime} \quad 3^{\prime \prime} 3$ $\delta 1873.0$ $-4045^{\prime} 27^{\prime \prime} 2$ » $17.5 \quad 11 \quad 3924.0 \quad-5 \quad 5622.5$ $\begin{array}{llllllll}7 & 26.5 & 17 & 2 & 21.3 & -7 & 1 & 35.1\end{array}$ Aug. 5.5 $\begin{array}{llllll}21 & 55 & 15.3 & -8 & 49 & 27.4\end{array}$

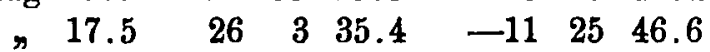

Nach der Methode der Variation der Distanzen erbielt ich aus denselben das folgende Elementensystem:

$$
\left.\begin{array}{rl}
T=1873 & \text { Juni } 25.38179 \text { mittl. Berl. Zeit. } \\
\pi & =3064^{\prime} 52^{\prime \prime} 6 \\
8 & =12054 \quad 40.8 \\
i & =124427.8
\end{array}\right\} \text { mittl. Aeq. } 1873.0
$$

\begin{tabular}{|c|c|c|c|c|}
\hline $\begin{array}{l}\text { 12h miltl. } \\
\text { Berl. Zeil. }\end{array}$ & $\alpha$ app. & $\delta$ app. & $\log \triangle$ & $\begin{array}{l}\text { Licht- } \\
\text { slärke }\end{array}$ \\
\hline 1873 Sept. 9 & $1^{\mathrm{h}} 53^{\mathrm{m}} 11^{\mathrm{s} .0}$ & $-16047^{\prime} 55^{\prime \prime}$ & 9.80466 & 0.86 \\
\hline 10 & $\begin{array}{lll}1 & 52 & 55.7\end{array}$ & $\begin{array}{lll}17 & 0 & 33\end{array}$ & & \\
\hline 11 & 15237.5 & $17 \quad 1254$ & & \\
\hline 12 & $\begin{array}{lll}152 & 16.4\end{array}$ & 172459 & & \\
\hline 13 & $\begin{array}{lll}15152.6\end{array}$ & 173647 & 9.80914 & 0.82 \\
\hline 14 & $\begin{array}{lll}1 & 5126.2\end{array}$ & $\begin{array}{lll}17 & 48 & 17\end{array}$ & & \\
\hline 15 & 15057.4 & $17 \quad 5928$ & & \\
\hline 16 & 15026.1 & $\begin{array}{lll}18 & 10 & 19\end{array}$ & & \\
\hline 17 & $\begin{array}{lll}149 & 52.4\end{array}$ & 182050 & 9.81498 & 0.77 \\
\hline 18 & $\begin{array}{lll}149.5 & 16\end{array}$ & $\begin{array}{lll}-18 & 30 & 59\end{array}$ & & \\
\hline
\end{tabular}

Bei Darstellung der mittleren Orte bleiben die folgenden Fehler übrig:

$$
\begin{aligned}
& \triangle \lambda \cos \beta=-8^{\prime \prime} 8,+3^{\prime \prime} 6,-0^{\prime \prime} 3 \\
& \triangle \beta=+2.1,-3.0,+2.5
\end{aligned}
$$

Zur weitern Verfolgung des Cometen gebe ich die

\begin{tabular}{|c|c|c|c|c|c|c|}
\hline $\begin{array}{l}\text { 12h mittll } \\
\text { Berl, Zei }\end{array}$ & & $\alpha$ app. & $\delta$ app & & $\log \Delta$ & $\begin{array}{l}\text { Licht- } \\
\text { stärke }\end{array}$ \\
\hline 373 Sept & 19 & $1^{b} 48^{m} 38^{s} .4$ & $-18040^{\prime}$ & $44^{\prime \prime}$ & & \\
\hline & 20 & $\begin{array}{lll}148.3 & 48.5\end{array}$ & 1850 & 6 & & \\
\hline & 21 & $\begin{array}{lll}147.3 & 47\end{array}$ & 1859 & 4 & 9.82228 & 0.72 \\
\hline & 22 & 14632.5 & 197 & 37 & & \\
\hline & 23 & 14547.0 & 1915 & 43 & & \\
\hline & 24 & $\begin{array}{lll}145 & 0.0\end{array}$ & 1923 & 23 & & \\
\hline & 25 & 14411.5 & 1930 & 36 & 9.83114 & 0.68 \\
\hline & 26 & $\begin{array}{lll}1 & 43 & 21.7\end{array}$ & 1937 & 21 & & \\
\hline & 27 & $\begin{array}{lll}142 & 30.8\end{array}$ & 1943 & 38 & & \\
\hline & 28 & 14138.9 & 1949 & 26 & & \\
\hline & 29 & 14046.0 & 1954 & 45 & 9.84160 & 0.63 \\
\hline & 30 & $\begin{array}{lll}1 & 39 & 52.3\end{array}$ & 1959 & 34 & & \\
\hline Oct. & 1 & 13858.1 & 203 & 54 & & \\
\hline & 2 & $138 \quad 3.4$ & 207 & 44 & & \\
\hline & 3 & $\begin{array}{lll}137 & 8.3\end{array}$ & 2011 & 3 & 9.85365 & 0.58 \\
\hline & 4 & $\begin{array}{lll}1 & 36 & 12.9\end{array}$ & 2013 & 52 & & \\
\hline & 5 & $\begin{array}{lll}135.3 & 17.3\end{array}$ & 2016 & 12 & & \\
\hline & 6 & 13421.6 & 2018 & 3 & & \\
\hline & 7 & 13325.8 & 2019 & 25 & 9.86723 & 0.53 \\
\hline & 8 & $\begin{array}{lll}132 & 30.2\end{array}$ & 2020 & 17 & & \\
\hline & 9 & 13135.0 & 2020 & 40 & & \\
\hline & 10 & $\begin{array}{lll}1 & 30 & 30.2\end{array}$ & 2020 & 34 & & \\
\hline & 11 & $\begin{array}{lll}129 & 45.8\end{array}$ & -2020 & 0 & 9.88226 & 4 \\
\hline
\end{tabular}
nachstehende Ephemeride:
Anzahl der Beobachtungen.

Clinton 2, Mailand 2, Neapel 2, Wien 3. Mailand 2, Wien 3.

Berlin 2, Mailand 1, Padua 2, Wien 3. Padua 2, Wien 3. Wien 2.
Als Einheit der Lichtstärke wurde diejenige der Entdeckung angenommen.

\title{
Beobachtung des von Paul Henry in Paris entdeckten Cometen.
}

$$
\text { Mittl. Wien. Zt. } \alpha \text { app. } \quad \text { 1. f. p. } \delta \text { app. } \quad \text { l. f. p. }
$$

1873 August $28 \quad 15^{\text {h }} 34 \mathrm{~m} 53 \mathrm{~s} .7 \quad 8$ h $1 \mathrm{~m} 58_{\mathrm{s} .62} \quad 8.920 n \quad+57049^{\prime} 14^{\prime \prime} .3 \quad 9.594$

Vergleichstern 1873.0. Wien. Mikrogl. 8h $0 \mathrm{~m} 10$ s. $39+57050^{\prime} 1^{\prime \prime} .4$.

Wien, den 30. August 1873.

L. Schulhof.

\section{Beobachtungen des von Paul Henry am 23. August entdeckten Cometen auf der Hamburger Sternwarte.} erhalten:

Von dem von Paul Henry am 23. August entdeckten Cometen habe ich bis jetzt folgende Positionen 


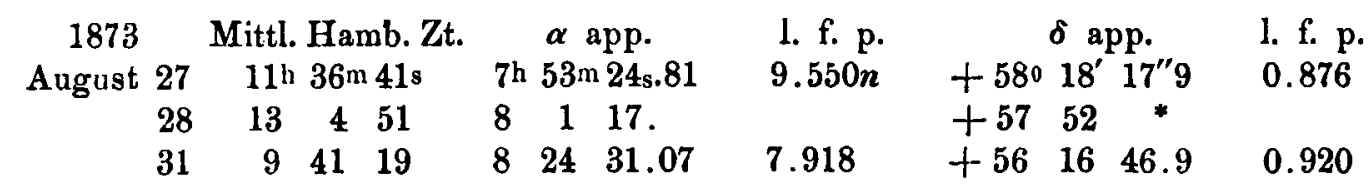

Comet sehr brillant mit einem sternartigen etwas nach Osten liegenden Kern; die Nebelmasse erscheint deutlich marmorirt. *Vergleichstern nicht bestimmt.

Comet Borelly (20. August).

$\begin{array}{rrrrrrrrrrrr}\text { August } 22 & 13 & 46 & 29 & \mathbf{7} & 29 & 36.96 & 9.606 n & +36 & \mathbf{4 8} & \mathbf{3 5 . 7} & 0.840 \\ 27 & 13 & 45 & 34 & \mathbf{7} & 36 & \mathbf{5 7 . 4 9} & \mathbf{9 . 5 8 2 n} & +31 & \mathbf{8} & 0.3 & 0.849 \\ 28 & 13 & 41 & 17 & \mathbf{7} & 38 & 31.45 & 9.575 n & +29 & \mathbf{5 2} & \mathbf{5 6 . 6} & 0.853\end{array}$

* Rund, 2' Durchmesser, verdichtet in der Mitte.

Comet Tempel.

$\begin{array}{lllllllllllll}\text { August } & 28 & 14 & 21 & 14 & 1 & 52 & 14.28 & 8.983 n & -14 & 4 & 39.2 & 0.909\end{array}$

Hamburger Sternwarte. September 1, 1873.

Comet schwach.

C. F. Pechiile.

\section{Aus einem Schreiben des Herrn Stephan, Directors der Sternwarte in Marseille, an den Herausgeber.}

J'ai l'honneur de vous transcrire à après nos premières observations de la planète (133) (Watson) et de la comète III 1873 (Borelly).

Observations de la planète (133) (Watson).

Date de l'obs. Heure (t.m.dn n. O.) AR. de (133) $\quad$ l. f. p. $\quad$ P. de (133) $\quad$ l. f. p. * de Comp. Obs.

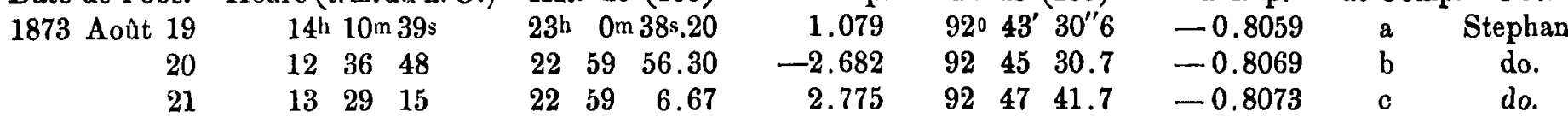

Pos. moy. des étoiles de comp. de (133) pour 1873.0:

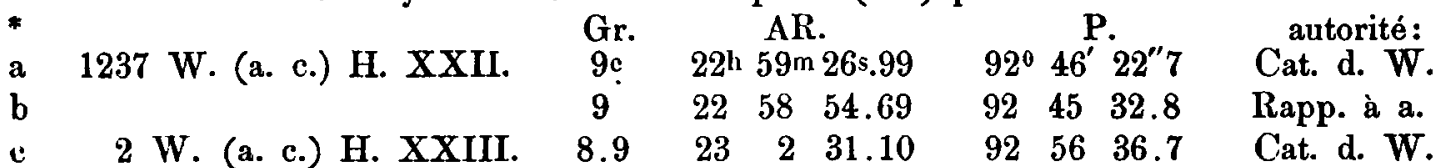

Observations de la comète c. 1873 (Borelly).

Date de l'obs. Heure(t.m.du n.O.) AR. de $\& \quad$ l. f. p. $\quad$ P. de $\&$ l. f. p. * de Comp. Obs.

1873 Août $20 \quad 15 \mathrm{~h} 43^{\mathrm{m} 40_{\mathrm{s}}} \quad$ 7h $27 \mathrm{~m} 1 \mathrm{s.5} \quad-1.744 \quad 51^{\prime \prime} 15^{\prime} 46^{\prime \prime} 5 \quad-0.6509 \quad$ a Borelly

$\begin{array}{llllllllllllll}21 & 14 & 54 & 14 & & 7 & 28 & 20.02 & -1.736 & 52 & 13 & 26.5 & -0.6299 & \text { b }\end{array}$

Pos. moy. des étoiles de comp. de la comète pour 1873.0:
*:
Gr.
$6 \mathrm{c}$
$8 \mathrm{c}$
Ak.
887 W. (n. c.) H. VII.
7h $31 \mathrm{~m} 41 \mathrm{~s} .82$
P.
b 664 W. (n. c.) H. VII.
$\begin{array}{lll}7 & 24 & 55.79\end{array}$
$51^{\prime \prime} 22^{\prime} 1^{\prime \prime} 6$
$52 \quad 24 \quad 14.6$
antorité :
Cat. $\mathrm{W}_{2}$.
Marseille, le 26. Aont 1873.

\section{In halt:}

(Zu No. 1955): Ueber das Meleor vom 17. Juni 1873. Von G. v. Niess/, Professor an der technischen Hochschule in Brünn. 161, - Elemente und Ephemeride des Brorsen'schen Comelen für seine Wiederkunft 1873. Von Dr. L. R. Schulze. 173

(Zu No. 1956): Beobar'htung des von Herrn Paul Hevry am 23. August enldecklen Coneten. Von Prof. Axel Möller. 183. - Schreiben des Herrn $\boldsymbol{W} \boldsymbol{E}$. Piziminer, Astronomen der Sternwarte des Herrn Bishop, an den Herausgelier. 183. - Cometen-Beobachtungen auf der Sternwarte in Lund Von Prof Axel Mislier. 185. - Beobachlung des Borelly'schen Cometen am Aequaloreal der Allonaer Sternwarle. Von Dr. C. F. W. Peters 185. - Elemente und Ephemeride des von Borelly in Marseille am 20. August entdecklen Cometen, bererhnel von dem c. M. Prof, E. Weiss. 187. - Elcmente und Ephemeride des Borelly'schen Cometen. Von Dr. C. F. W. Peters. 187. - Elemente und Epheneride des Cometen II. 1573. Von Dr. L. Schulhof. 187. - Beobachtungen des von Paul He „ry am 23 Ausust entdeckten Conelen aul der Hambur, de's Herru Stephan, Directors der Slernwarle in Marseille, an den Heransgeber. 191. 